\title{
Managing Cardiac Disease in Pregnancy
}

\section{Reference: British Journal of Midwifery, November 2005, Vol 13, N11 pages 1-3}

\begin{abstract}
More women with congenital heart disease now reach their adult reproductive years in good health. It is likely therefore that the number of those who present for care during pregnancy will continue to increase. The physiological changes that normally occur in pregnancy can compromise the conditions of women with heart disease, often leaving them with minimal cardiovascular reserve. There are particularly high risks associated with certain cardiac conditions and pregnancy in these women is contraindicated. Women with congenital heart disease can be physically very ill in pregnancy and most have added emotional requirements. The needs of these women are often complex and diverse with many potential complications. Midwives caring for these patients are consequently challenged to remain not only competent in midwifery, but also in many aspects of cardiology, together with the need to remain sensitive to the emotional care requirements that accompany these conditions.
\end{abstract}

\section{Introduction}

The past few decades have heralded many wonderful accomplishments and innovations in the management of cardiac disease. Children with complex congenital cardiac anomalies now have increased life expectancies and as a result many reach their adult reproductive years in good health. These exciting developments challenge midwives to remain abreast with on-going developments in both midwifery and cardiology. According to Iserin (2001) women with congenital heart disease represents the majority of those with heart disease who present for care during 
pregnancy. It is likely therefore that in the future increasing numbers of women with congenital heart disease will present for midwifery care. While the majority of women with heart disease have successful pregnancies and cardiac complications during pregnancy are rare, there are some conditions that pose significant challenges to midwives, obstetricians and cardiologists, particularly when the pregnancy threatens the life or health of the mother or baby. This article outlines many of the pertinent and current issues for consideration by midwives who encounter women with congenital cardiac disease in pregnancy.

\section{Cardiovascular Disease in Pregnancy}

Cardiovascular disease in pregnancy is becoming increasingly evident, as women with congenital heart disease (CHD) have longer life-expectancies than previously. Scientific advances in medical care in recent years have resulted in survival rates of up to $85 \%$ of people with congenital heart disease (Swinburne, 2004). It is estimated that there are between 16,000 and 20,000 people in the United Kingdom with congenital heart disease. This number is expected to increase at a rate of about 2,000 per year (Swinburne, 2004). Most women with heart disease can have successful pregnancies and cardiac complications during pregnancy tend to be rare. Women with serious cardiac disease generally carry the highest maternal mortality risk, irrespective of their underlying cardiac condition. Those women with less serious underlying cardiac diseases tend to have a higher rate of successful pregnancies.

\section{Physiological Changes in Pregnancy}


As a prelude to discussing cardiac disease during pregnancy, it is important to recall the main haemodynamic modifications that occur during this time.

The physiological changes that occur during pregnancy are similar to the adaptations that occur in the cardiovascular and respiratory systems during exercise (Martens et al, 2006). As a result of hormonal changes there is an increase in blood volume, which starts as early as the fifth week. By the end of pregnancy, cardiac output can increase by $30-50 \%$, due to increases in stroke volume and heart rate (Jarski and Trippett, 1990). Diastolic blood pressure falls and there is a reduction in systemic vascular resistance (Reimold and Rutherford, 2003). There is also an increased risk of thromboembolism during pregnancy, with the risk being highest after delivery (Lee, 2005). In the post partum period these physiological changes may not normalize for several weeks (Iserin, 2001).

\section{Cardiac Conditions in Pregnancy}

Those haemodynamic changes which occur normally in pregnancy can exacerbate the problems associated with serious heart disease, as women with heart disease are left with little or no remaining cardiovascular reserve. The changes in intravascular volume, cardiac output and systemic vascular resistance can cause maternal or fetal deterioration in women with pre-existing congenital heart disease (Siu et al, 1999). Atrial-septal defect is the most prevalent form of congenital heart disease in adults. However, this condition barely poses any complications in pregnancy unless associated with pulmonary hypertension (Zuber et al, 1999). All women with clinically significant valvular heart disease should ideally be assessed prior to 
conception and this should include a full medical history along with a detailed cardiac assessment (Reimold and Rutherford, 2003). Conversely, Siu et al (1999) state that many women with congenital heart disease reach adulthood without receiving any genetic assessment or counselling about the cause, inheritance, recurrence risk and prenatal diagnosis options available to them.

There are some cardiac conditions that render women with CHD at particularly high risk. These conditions include;

- $\quad$ Severe pulmonary vascular disease: This includes patients with or without septal defects. These patients are known to carry the highest maternal mortality risk (30-50\%) (Weiss and Hess, 2000). There is an additional increase in pulmonary vascular resistance, which is life-threatening when associated with these conditions. This develops rapidly in the peripartum and postpartum periods. There is right to left shunting during pregnancy in patients with Eisenmenger syndrome because of systemic vasodilation and right ventricular overload. This causes increased cyanosis and decreased pulmonary perfusion (Oakley et al, 2003). Recommendations for patients with Eisenmenger syndrome include avoidance of vasodilatation, maintenance of preload and the use of prophylactic heparin (Oakley, 1997).

- Severe left ventricular outflow obstruction: Severe aortic stenosis also carries a high risk. In women with this condition the outflow tract 
resistance is unable to accommodate the increased cardiac output caused by increased plasma volume, which can cause heart failure, low cardiac output and pulmonary congestion.

- Cyanotic heart disease: There is a high risk of complications associated with this condition, including a 50\% risk of spontaneous abortion, a risk of premature delivery and low birth weight babies, as maternal hypoxaemia impairs fetal growth (Oakley et al, 2003). There is about a 2\% maternal mortality rate and a poor fetal prognosis.

Pregnancy is not recommended in the aforementioned high-risk group of patients. As the risk to the mother is so high, termination is often most recommended (Oakley et al, 2003). Midwives caring for these patients are challenged with being highly competent in delivering complex care, as many of these patients can be very ill as well as having significant emotional requirements. Thromboembolism is a major risk factor in high-risk pregnancies. The midwife must be vigilant in detecting this potential complication, particularly following caesarean section and during the puerperium.

\section{On-going Pregnancy in High-Risk Patients}

It is important that pregnant women with severe cardiac disease are made aware of the implications of pregnancy for themselves and the fetus. Once an informed choice has been made and if the pregnancy is not terminated then it is the role of the midwife to 
offer maximum support to the woman in her decision and to provide evidence-based midwifery practice. This will include collaborative care with the cardiologist, obstetrician, neonatologist and other members of the multidisciplinary team, depending on the individuals identified needs. The woman must be advised to restrict physical activity and take as much rest as possible. It is recommended that the woman be hospitalised by the end of the second trimester and anticoagulants should be administered subcutaneously, as a prophylactic measure against thromboembolism (Oakley et al, 2003). Where patients present with severe aortic stenosis, the midwife must remain vigilant for signs of left ventricular overload. Monitoring of systemic blood pressure and ECG are important features, which can provide the midwife with early indicators of a potentially deteriorating condition. The monitoring of oxygen saturations is especially important in patients with severe cyanotic heart disease. According to Oakely et al. (2003) haematocrit and haemoglobin levels are inaccurate indicators, as haemodilution occurs in pregnancy.

\section{Pregnancy in Low-Risk Patients}

Women with less serious cardiac conditions generally tend to tolerate pregnancy well. Interestingly, women with small to moderate shunts or mild to moderate valve regurgitation tend to benefit from the decrease in systemic vascular resistance that occurs during pregnancy.

- Women with prosthetic heart valves generally tolerate the haemodynamic consequences of pregnancy. There is however an increased risk of thrombosis and a high risk of thromboembolic complications because of the 
hypercoagulable status of the pregnant woman (Prasad and Ventura, 2001). There is an absolute need for anticoagulation therapy with mechanical prostheses. Vitamin $\mathrm{K}$ antagonists cross the placenta so there is a small increased risk $(<5 \%)$ of early abortion, embroyopathy and premature delivery (Oakely et al, 2003).

- Young women who have had successful cardiac surgery, including correction of tetralogy of fallots and fontan repair, generally tolerate pregnancy quite well, provided their left ventricular function is good. It is recommended that patients with complex congenital defects discuss their preconceptual intentions with their cardiac physician to ensure that individual risks can be fully discussed. Midwives should use all available opportunities to educate women of these recommendations and the associated benefits.

Specialist monitoring provided by both the obstetric and cardiology services is warranted for all pregnant patients with cardiac disease.

\section{Dysrhythmias in pregnancy}

The incidence of cardiac dysrhythmias, arising from either the artia or ventricles, increases in pregnancy and affects up to $80 \%$ of patients with congenital heart disease. The cause is linked with hormonal, emotional and haemodynamic changes (Tan and Lee, 2001). While digoxin has traditionally been the drug of choice for the management of chronic dysrhythmias, is has been found to be ineffective (Oakely et al, 2003). Amiodarone, which has many side effects, including fetal hypothyroidism, 
should be avoided where possible during pregnancy and if administration is necessary, then the lowest possible dose to achieve efficacy should be given. Antiarrhythmics such as verapmil, quinidine and beta-blockers are more suitable drug choices and have not been associated with teratogenic effects (Bloomfield and Hawkins, 1991). Episodes of sustained tachycardia that are poorly tolerated by the mother can cause hypoperfusion to the fetus, causing negative effects. It is advisable that drug therapy be initiated even if the tachycardia is tolerated by the woman and if not, a decision should be made to cardiovert the patient with the aim of restoring sinus rhythm.

\section{The Fetus}

There is a $2-16 \%$ risk of congenital heart disease to the fetus of a woman with congenital heart disease. It is necessary therefore that fetal cardiac assessment be performed. The incidence of CHD in the fetus is higher when it is the mother rather than the father who is affected by the condition. In situations where the fetus is affected by the condition, it is advisable for the delivery to take place in a tertiary centre. There is a high survival rate for pre-term neonates delivered after 32 weeks gestation. Delivery should be postponed as long as possible up to 32 weeks, at which time decisions about delivery will based on maternal and fetal wellbeing and fetal growth (Oakely et al, 2003). 


\section{Advice and Information}

Iserin (2001) outlines some issues to be considered for women with congenital heart disease who present for pre-conceptual care. These include the need for an accurate medical and cardiac assessment, the provision of as much information as possible about the anticipated risks to the mother and fetus and the identification of other associated or non-associated risk factors that may pose problems in the pregnancy. It is also advisable to encourage pregnancy early in life.

During the pregnancy the following information may be of assistance:

- The administration of high doses of steroids, for pulmonary maturation, is safe but can induce volume expansion, which can place additional strain on the heart.

- The use of oxytocin or prostaglandin to induce labour can cause bradycardia, hypertension, myocardial ischaemia and vasospasm.

- The risk of causing cardiac failure from the administration of volume expanding solutions must be balanced against the benefits of providing spinal anaesthesia in labour.

\section{Conclusion}


Heart disease in young pregnant women was rare until recent years but is something that will be increasingly witnessed, as patients with complex congenital cardiac anomalies live longer. The changes that typically arise in pregnancy can exacerbate pre-existing problems for women with serious heart disease, as they are sometimes left with little or no remaining cardiac reserve. Preconceptual advice should be sought by and provided for all young women who have known cardiac disease. This will facilitate individual risk assessment and the provision of important information. Pregnancy is not recommended in women who present with high-risk cardiac diseases. However, women who elect to become or remain pregnant require considerable psychological support and evidence-based midwifery care, which is often complex. Midwives are therefore challenged with remaining abreast with midwifery and aspects of specialist cardiology. This article outlined some of the clinical issues to be considered by midwives who face these challenges

\section{References}

Bloomfield T, Hawkins D (1991) The effects of drugs on the human fetus. In: Setchell and Ginsberg eds. Scientific Foundations of Obstetrics and Gynaecology. Butterworth-Heinemann, Oxford: 320-336.

Iserin L (2001) Management of pregnancy in women with congenital heart disease. Heart 85(5):493-494.

Jarski R, Trippett D (1990) The risks and benefits of exercise during pregnancy. Journal of Family Practice 30, 185-190.

Lee R (2005) Thromboembolism in pregnancy: a continuing conundrum. Annals 
of Internal Medicine 43(10):749-750.

Martens D, Hernandez B, Strickland G, Boatwright D (2006) Pregnancy and Exercise: Physiological Changes and Effects on the Mother and Fetus. Strength and Conditioning Journal 28(1):78-82

Oakley C (1997) Pregnancy and congenital heart disease. Heart 8, 12-14.

Oakley C, Child A, Lung B (2003) Expert consensus document on management of cardiovascular diseases during pregnancy. European Heart Journal 24, 761781.

Prasad A, Ventura H (2001) Valvular heart disease and pregnancy: a high index of suspicion important to reduce risks. Postgraduate Medicine 110(2): 69-79.

Reimold S, Rutherford J (2003) Valvular heart disease in pregnancy. The New England Journal of Medicine 349(1):52-60.

Siu S, Chitayat D, Webb G (1999) Pregnancy in women with congenital heart defects: what are the risks? Heart 81, 225-226.

Swinburne C (2004) Take Heart. Nursing Standard 18(42): 18-19.

Tan H, Lee K (2001) Treatment of tachyarrhythmias during pregnancy and lactation. European Heart Journal 22, 458-464.

Weiss B, Hess O (2000) Pulmonsry vascular disease and pregnancy: current controversies, management and perspectives. European Heart Journal 21, 10405 .

Zuber M, Gautschi N, Oechslin E, Widmer V, Kiowski W, Jennu R (1999) Outcome of pregnancy in women with congenital shunt lesions. Heart 81, 271-275. 
